\title{
Noncommutative Supertori in Two Dimensions
}

\author{
Ee Chang-Young1 \\ Department of Physics, Sejong University, Seoul 143-747, Korea \\ HoIL KIM2 \\ Department of Mathematics, Kyungpook National University, \\ Taegu 702-701, Korea \\ and \\ HIROAKI NAKAJIMA 3 \\ Department of Physics and Institute of Basic Science, \\ Sungkyunkwan University, Suwon 440-746, Korea
}

\begin{abstract}
First we consider the deformations of superspaces with $\mathcal{N}=(1,1)$ and $\mathcal{N}=(2,2)$ supersymmetries in two dimensions. Among these the construction of noncommutative supertorus with odd spin structure is possible only in the case of $\mathcal{N}=(2,2)$ supersymmetry broken down to $\mathcal{N}=(1,1)$. However, for the even spin structures the construction of noncommutative supertorus is possible for both $\mathcal{N}=(1,1)$ and $\mathcal{N}=(2,2)$ cases. The spin structures are realized by implementing the translational properties along the cycles of commutative supertorus in the operator version: Odd spin structure is realized by the translation in the fermionic direction in the same manner as in the construction of noncommutative torus, and even spin structures are realized with appropriate versions of the spin angular momentum operator.
\end{abstract}

PACS: 02.40.Gh 11.30.Pb

\footnotetext{
${ }^{1}$ cylee@sejong.ac.kr

${ }^{2}$ hikim@knu.ac.kr

${ }^{3}$ nakajima@skku.edu
} 


\section{Introduction}

Ever since the work of Connes, Douglas, and Schwarz [1] on the toroidal compactification of M(atrix) theory using the concept of noncommutative geometry [2], the noncommutative torus and noncommutative geometry in general has become a household subject in string theory [3, 4].

Noncommutative geometry naturally appears in string theory: Low-energy effective theory of D-branes in a background NSNS $B$-field becomes the noncommutative field theory where the spacetime coordinates $x^{\mu}$ are noncommutative, $\left[x^{\mu}, x^{\nu}\right] \neq 0$ [1, 5, 6]. If we turn on the background RR field, the low-energy effective theory of D-branes becomes the field theory on non(anti)commutative superspace of which the fermionic coordinate $\theta^{\alpha}$ has nontrivial commutation relation $\left\{\theta^{\alpha}, \theta^{\beta}\right\} \neq 0[7,8,9,10,11]$. Gauge theories on non(anti)commutative superspace are studied extensively [12, 13, 14, 15].

Toroidal compactification in string theory with the above mentioned background fields then naturally leads to noncommutative supertorus. Although the noncommutative torus is a very well known subject, its supersymmetric version, the noncommutative supertorus, still remains virtually unknown. Commutative supertorus was constructed by Rabin and Freund [16] based on the work of Crane and Rabin [17] on super Riemann surfaces. The supertorus was obtained as the quotient of superplane by a subgroup of $\operatorname{Osp}(1 \mid 2)$ which acts properly discontinuously on the plane together with the metrizable condition. These two conditions boil down to proper latticing of the superplane, and can be expressed as appropriate translation properties along the cycles of the torus.

In this paper, adapting the guideline of defining noncommutative torus we construct noncommutative supertorus. Noting that the construction of bosonic noncommutative torus is guided by the classical translation properties of the commutative torus along its cycles, we construct the noncommutative supertorus with the following two guidelines: Express the translations along the cycles of the supertorus in the operator language, and implement the spin structures of supertorus for even and odd translations with the spin angular momentum operator in appropriate representations. Noncommutative torus is defined by embedding the lattice [18, 19, 20, 21] into the Heisenberg group [22, 23, 24]. The lattice embedding determines how the generators of noncommutative torus, which correspond to the translation operators along the cycles of the commutative torus, would act on the module of the noncommutative torus. The Heisenberg group can be regarded as a central extension of commutative space, which is equivalent to a deformation of space by constant noncommutativity. Recently, we constructed the super Heisenberg group [25] as a central extension of ordinary superspace, which is equivalent to the deformation of superspace by constant 
noncommutativity and nonanticommutativity. Based on our construction of super Heisenberg group, we define the embedding maps for noncommutative supertori in two dimensions. Incorporating the spin structures is an additional task implementing the translational properties along the cycles of commutative supertorus in the operator language. For the odd spin structure case it is realized by the translation in the fermionic direction as in the construction of noncommutative torus. For the even spin structure cases, they are realized with appropriate representations of the spin angular momentum operator.

This paper is organized as follows. In section 2, we briefly recall the definition of noncommutative torus and the projective module on which it acts. Construction of the noncommutative tori via embedding the lattice into Heisenberg group is also explained in the Heisenberg representation. In section 3, we briefly review the commutative supertori. In section 4, we first recall the deformation of superspace in relation with super Heisenberg group. Then, we explicitly perform the construction of noncommutative supertorus via embedding map in the cases of $\mathcal{N}=(1,1)$ and $\mathcal{N}=(2,2)$. We conclude in section 5 .

\section{Noncommutative tori}

Noncommutative torus $\left(\mathbb{T}_{\theta}^{d}\right)$ is an algebra defined by generators $U_{1}, \cdots, U_{d}$ obeying the following relations:

$$
U_{i} U_{j}=e^{2 \pi i \theta_{i j}} U_{j} U_{i}, \quad i, j=1, \cdots, d,
$$

where $\left(\theta_{i j}\right)$ is a real $d \times d$ anti-symmetric matrix. $\mathbb{T}_{\theta}^{d}$ defines the involutive algebra

$$
\mathcal{A}_{\theta}^{d}=\left\{\sum a_{i_{1} \cdots i_{d}} U_{1}^{i_{1}} \cdots U_{d}^{i_{d}} \mid a=\left(a_{i_{1} \cdots i_{d}}\right) \in \mathcal{S}\left(\mathbb{Z}^{d}\right)\right\}
$$

where $\mathcal{S}\left(\mathbb{Z}^{d}\right)$ is the Schwartz space of sequences with rapid decay.

Every projective module over a smooth algebra $\mathcal{A}_{\theta}^{d}$ can be represented by a direct sum of modules of the form $\mathcal{S}\left(\mathbb{R}^{p} \times \mathbb{Z}^{q} \times F\right)\left[18\right.$, the linear space of Schwartz functions on $\mathbb{R}^{p} \times \mathbb{Z}^{q} \times F$, where $2 p+q=d$ and $F$ is a finite abelian group. Let $D$ be a lattice in $\mathcal{G}=M \times \widehat{M}$, where $M=\mathbb{R}^{p} \times \mathbb{Z}^{q} \times F$ and $\widehat{M}$ is its dual. The embedding map $\Phi$ under which $D$ is the image of $\mathbb{Z}^{d}$ determines a projective module $E$ on which the algebra of the noncommutative torus acts.

In the Heisenberg representation the operators U's are defined by

$$
U_{(m, \hat{s})} f(r)=e^{2 \pi i<r, \hat{s}>} f(r+m), \quad m, r \in M, \hat{s} \in \widehat{M}, f \in E,
$$


where $<r, \hat{s}>$ is a usual inner product between $M$ and $\widehat{M}$. Here, the vector $(m, \hat{s})$ can be mapped into an element of the Heisenberg group which we explain next.

The Heisenberg group, Heis $\left(\mathbb{R}^{2 n}, \psi\right)$, is defined as follows. For $t, t^{\prime} \in U(1)$, and $(x, y),\left(x^{\prime}, y^{\prime}\right) \in$ $\mathbb{R}^{2 n}$, we define the product for $(t, x, y),\left(t^{\prime}, x^{\prime}, y^{\prime}\right) \in \operatorname{Heis}\left(\mathbb{R}^{2 n}, \psi\right)$,

$$
(t, x, y) \cdot\left(t^{\prime}, x^{\prime}, y^{\prime}\right)=\left(t+t^{\prime}+\psi\left(x, y ; x^{\prime}, y^{\prime}\right), x+x^{\prime}, y+y^{\prime}\right)
$$

where $\psi: \mathbb{R}^{2 n} \times \mathbb{R}^{2 n} \longrightarrow \mathbb{R}$, satisfies the cocycle condition

$$
\psi\left(x, y ; x^{\prime}, y^{\prime}\right) \psi\left(x+x^{\prime}, y+y^{\prime} ; x^{\prime \prime}, y^{\prime \prime}\right)=\psi\left(x, y ; x^{\prime}+x^{\prime \prime}, y^{\prime}+y^{\prime \prime}\right) \psi\left(x^{\prime}, y^{\prime} ; x^{\prime \prime}, y^{\prime \prime}\right)
$$

which is a necessary and sufficient condition for the multiplication to be associative. There is an exact sequence

$$
0 \rightarrow \mathbb{R} \stackrel{i}{\rightarrow} \operatorname{Heis}\left(\mathbb{R}^{2 n}, \psi\right) \stackrel{j}{\rightarrow} \mathbb{R}^{2 n} \rightarrow 0
$$

called a central extension, with the inclusion $i(t)=(t, 0,0)$ and the projection $j(t, x, y)=$ $(x, y)$, where $i(\mathbb{R})$ is the center in $\operatorname{Heis}\left(\mathbb{R}^{2 n}, \psi\right)$. The previously appeared vector $(m, \hat{s})$ in (3) corresponds to a vector $(x, y) \in \mathbb{R}^{2 n}$ in the above description of the Heisenberg group.

Now, we consider an explicit form of the embedding in a typical case where $M$ in (3) is given by $M=\mathbb{R}^{p}$. In this case, one can define the embedding map in the canonical form as

$$
\Phi=\left(\begin{array}{cc}
\Theta & 0 \\
0 & I
\end{array}\right):=\left(x_{i, j}\right), \text { where } \Theta=\operatorname{diag}\left(\theta_{1}, \cdots, \theta_{p}\right), \quad i, j=1, \cdots, 2 p,
$$

then the Heisenberg representation is given as follows.

$$
\left(U_{j} f\right)\left(s_{1}, \cdots, s_{p}\right):=\left(U_{\vec{e}_{j}} f\right)(\vec{s})=\exp \left(2 \pi i \sum_{k=1}^{p} s_{k} x_{k+p, j}\right) f\left(\vec{s}+\vec{x}_{j}\right), \quad \text { for } \quad j=1, \cdots, 2 p,
$$

where $\vec{e}_{j}=\left(x_{1, j}, \ldots, x_{2 p, j}\right)$. The above can be redisplayed as

$$
\left(U_{j} f\right)(\vec{s})=f(\vec{s}+\vec{\theta}), \quad\left(U_{j+p} f\right)(\vec{s})=e^{2 \pi i s_{j}} f(\vec{s}), \quad j, k=1, \cdots, p,
$$

with $\vec{s}=\left(s_{1}, \cdots, s_{p}\right), \vec{x}_{j}=\left(x_{1, j}, \cdots, x_{p, j}\right)$ and $\vec{s}, \vec{x}_{j} \in \mathbb{R}^{p}$. Here, $U_{j}$ 's satisfy

$$
U_{j} U_{j+p}=e^{2 \pi i \theta_{j}} U_{j+p} U_{j}, \text { otherwise } U_{j} U_{k}=U_{k} U_{j} .
$$

In the general embedding case, we will use the Manin's representation [20] in which (8) becomes

$$
\left(U_{\vec{e}_{j}} f\right)(\vec{s}):=\exp \left(2 \pi i \sum_{k=1}^{p} s_{k} x_{k+p, j}+\frac{1}{2} \sum_{k=1}^{p} x_{k, j} x_{k+p, j}\right) f\left(\vec{s}+\vec{x}_{j}\right), \quad \text { for } \quad j=1, \cdots, 2 p .
$$

This representation corresponds to the second representation of the Heisenberg group in [25]. 


\section{Commutative supertori}

A two-dimensional commutative torus is given by $\mathbb{T}^{2}=\mathbb{C} /(\mathbb{Z}+\tau \mathbb{Z})$, where $\tau$ is a complex structure. In a similar way, as the result of the supersymmetric version of the uniformization theorem [17], a supertorus is given by a quotient of the two-dimensional superspace by a subgroup of $\operatorname{Osp}(\mathcal{N} \mid 2)$ which is the anomaly-free part of the superconformal group [17, 16]. The action of this subgroup gives the cycles of the supertorus. When we consider $\mathcal{N}=$ $(1,1)$ superspace spanned by supercoordinates $(z, \bar{z}, \theta, \bar{\theta})$ as an ambient space, the action of $\operatorname{Osp}(1 \mid 2)$ in the holomorphic sector is given by

$$
\begin{aligned}
& z \rightarrow z^{\prime}=\frac{a z+b}{c z+d}+\theta \frac{\gamma z+\delta}{(c z+d)^{2}}, \quad a d-b c=1, \\
& \theta \rightarrow \theta^{\prime}=\frac{\gamma z+\delta}{c z+d}+\frac{\theta}{c z+d}\left(1+\frac{1}{2} \delta \gamma\right) .
\end{aligned}
$$

The condition for the subgroup is i) the supertorus is metrizable, i.e. the metric of the supertorus is invariant under this subgroup, ii) this subgroup acts properly discontinuously, which means that $z$ and $z^{\prime}$ have disjointed neighborhoods. From these conditions, we have $c=\gamma=0$ and (12) is reduced to

$$
\begin{aligned}
& z \rightarrow z^{\prime}=a^{2} z+a b+a^{2} \theta \delta, \\
& \theta \rightarrow \theta^{\prime}=a(\theta+\delta),
\end{aligned}
$$

where $a= \pm 1$. The sign of $a$ in each cycle determines the spin structure. The action of the subgroup (13) is more simplified by the similarity transformation via an element of Osp $(1 \mid 2)$. Then it is classified to the four cases according to the spin structure of the supertorus as follows:

- $(+,+)$ structure

$$
(z, \theta) \rightarrow(z+1, \theta), \quad(z, \theta) \rightarrow(z+\tau+\theta \delta, \theta+\delta)
$$

- $(+,-)$ structure

$$
(z, \theta) \rightarrow(z+1, \theta), \quad(z, \theta) \rightarrow(z+\tau,-\theta)
$$

- $(-,+)$ structure

$$
(z, \theta) \rightarrow(z+1,-\theta), \quad(z, \theta) \rightarrow(z+\tau, \theta) .
$$


- $(-,-)$ structure

$$
(z, \theta) \rightarrow(z+1,-\theta), \quad(z, \theta) \rightarrow(z+\tau,-\theta)
$$

Here $\tau$ and $\delta$ are the moduli parameters of the supertorus. The action of the subgroup (14)(17) for the anti-holomorphic sector $(\bar{z}, \bar{\theta})$ is obtained by complex conjugation. The $(+,+)$ structure is also called the odd spin structure and the other three structures are called the even spin structures.

$\mathcal{N}=(2,2)$ supertorus can be also constructed in a similar manner. Let $\left(z, \bar{z}, \theta^{ \pm}, \bar{\theta}^{ \pm}\right)$be supercoordinates of $\mathcal{N}=(2,2)$ superspace. Then the cycles in $\mathcal{N}=(2,2)$ supertorus with the odd spin structure are given by

$$
\begin{aligned}
& \left(z, \theta^{+}, \bar{\theta}^{+}\right) \rightarrow\left(z+1, \theta^{+}, \bar{\theta}^{+}\right) \\
& \left(z, \theta^{+}, \bar{\theta}^{+}\right) \rightarrow\left(z+\tau+\bar{\theta}^{+} \delta^{+}+\theta^{+} \bar{\delta}^{+}, \theta^{+}+\delta^{+}, \bar{\theta}^{+}+\bar{\delta}^{+}\right) .
\end{aligned}
$$

In the case of the $(+,-)$ structure, the cycles in the supertorus are given by

$$
\left(z, \theta^{+}, \bar{\theta}^{+}\right) \rightarrow\left(z+1, \theta^{+}, \bar{\theta}^{+}\right), \quad\left(z, \theta^{+}, \bar{\theta}^{+}\right) \rightarrow\left(z+\tau,-\theta^{+},-\bar{\theta}^{+}\right)
$$

The cycles in the other even spin structures are also obtained similarly.

\section{Noncommutative supertori}

Noncommutative supertorus is defined by embedding the lattice to the super Heisenberg group. The super Heisenberg group is given by the central extension of ordinary superspace, which is equivalent to the deformation of superspace by constant noncommutativity and nonanticommutativity.

\subsection{Deformation of superspace}

First we consider the deformation of $\mathcal{N}=(1,1)$ superspace spanned by supercoordinates $\left(X^{1}, X^{2}, \theta, \bar{\theta}\right)$. Supercharges and supercovariant derivatives are defined by

$$
\begin{aligned}
Q & =\frac{\partial}{\partial \theta}-\theta \frac{\partial}{\partial Z}, & \bar{Q} & =\frac{\partial}{\partial \bar{\theta}}-\bar{\theta} \frac{\partial}{\partial \bar{Z}} \\
D & =\frac{\partial}{\partial \theta}+\theta \frac{\partial}{\partial Z}, & \bar{D} & =\frac{\partial}{\partial \bar{\theta}}+\bar{\theta} \frac{\partial}{\partial \bar{Z}}
\end{aligned}
$$


where $Z, \bar{Z}$ are the complex coordinates given by

$$
\begin{aligned}
Z & =X^{1}+i X^{2}, & \bar{Z} & =X^{1}-i X^{2} \\
\frac{\partial}{\partial Z} & =\frac{1}{2}\left(\frac{\partial}{\partial X^{1}}-i \frac{\partial}{\partial X^{2}}\right), & \frac{\partial}{\partial \bar{Z}} & =\frac{1}{2}\left(\frac{\partial}{\partial X^{1}}+i \frac{\partial}{\partial X^{2}}\right) .
\end{aligned}
$$

Now we try to introduce the deformation for fermionic coordinates keeping the reality condition and preserving full or partial supersymmetry. However it turns out that it is impossible to perform the above deformation preserving the Heisenberg group structure. For instance, consider the Moyal product of the form,

$$
*^{\prime}=\exp \left[\frac{i}{2} \Theta \epsilon^{\mu \nu} \overleftarrow{\frac{\partial}{\partial X^{\mu}}} \frac{\partial}{\partial X^{\nu}}-\frac{C}{2}(\overleftarrow{Q} \vec{Q}+\overleftarrow{\bar{Q}} \vec{Q})\right]
$$

Then supersymmetry is completely broken, since under (24) the Leibniz rule

$$
Q\left(f *^{\prime} g\right)=(Q f) *^{\prime} g+(-1)^{|f|} f *^{\prime}(Q g), \quad \bar{Q}\left(f *^{\prime} g\right)=(\bar{Q} f) *^{\prime} g+(-1)^{|f|} f *^{\prime}(\bar{Q} g)
$$

is not satisfied, where $|f|$ is zero for Grassmann even $f$ and unity for Grassmann odd $f$. The next candidate of the Moyal product is

$$
*^{\prime \prime}=\exp \left[\frac{i}{2} \Theta \epsilon^{\mu \nu} \frac{\overleftarrow{\partial}}{\partial X^{\mu}} \frac{\partial}{\partial X^{\nu}}-\frac{C^{\prime}}{2}(\overleftarrow{D} \vec{D}+\overleftarrow{D} \vec{D})\right]
$$

In this case supersymmetry is preserved since (25) holds but the corresponding algebra in the operator formalism becomes

$$
\begin{aligned}
& {\left[X^{1}, X^{2}\right]=i\left[\Theta+\frac{C^{\prime}}{4}(\bar{\theta} \theta-\theta \bar{\theta})\right], \quad\left[X^{1}, \theta\right]=-\frac{C^{\prime}}{2} \bar{\theta}, \quad\left[X^{1}, \bar{\theta}\right]=-\frac{C^{\prime}}{2} \theta} \\
& {\left[X^{2}, \theta\right]=-\frac{i C^{\prime}}{2} \bar{\theta}, \quad\left[X^{2}, \bar{\theta}\right]=-\frac{i C^{\prime}}{2} \theta, \quad\{\theta, \bar{\theta}\}=C^{\prime},}
\end{aligned}
$$

which is not a super Heisenberg algebra since the commutator and anticommutator do not belong to the center. In order to obtain a super Heisenberg algebra, the parameter $C^{\prime}$ should vanish. Thus we consider the case of $C^{\prime}=0$ and use the following Moyal product

$$
*=\exp \left(\frac{i}{2} \Theta \epsilon^{\mu \nu} \overleftarrow{\frac{\partial}{\partial X^{\mu}}} \frac{\partial}{\partial X^{\nu}}\right)
$$

The corresponding algebra becomes

$$
\left[X^{1}, X^{2}\right]=i \Theta, \quad \text { others }=0,
$$


which can be represented as the action on the module $f(s, \theta, \bar{\theta})$ as follows.

$$
X^{1} f(s, \theta, \bar{\theta})=i \frac{\partial}{\partial s} f(s, \theta, \bar{\theta}), \quad X^{2} f(s, \theta, \bar{\theta})=s f(s, \theta, \bar{\theta}),
$$

where we set $\Theta=1$ by change of normalization of the operators.

Next we consider $\mathcal{N}=(2,2)$ superspace spanned by supercoordinates $\left(X^{1}, X^{2}, \theta^{ \pm}, \bar{\theta}^{ \pm}\right)$. Supercharges and supercovariant derivatives on $\mathcal{N}=(2,2)$ superspace are defined by

$$
\begin{array}{ll}
Q_{+}=\frac{\partial}{\partial \theta^{+}}-\bar{\theta}^{+} \frac{\partial}{\partial Z}, & \bar{Q}_{+}=\frac{\partial}{\partial \bar{\theta}^{+}}-\theta^{+} \frac{\partial}{\partial Z} \\
D_{+}=\frac{\partial}{\partial \theta^{+}}+\bar{\theta}^{+} \frac{\partial}{\partial Z}, & \bar{D}_{+}=\frac{\partial}{\partial \bar{\theta}^{+}}+\theta^{+} \frac{\partial}{\partial Z},
\end{array}
$$

and $\left(Q_{-}, \bar{Q}_{-}, D_{-}, \bar{D}_{-}\right)$which are obtained by the complex conjugation $Z \leftrightarrow \bar{Z}, \theta^{+} \leftrightarrow \theta^{-}$, $\bar{\theta}^{+} \leftrightarrow \bar{\theta}^{-}$. The deformation of $\mathcal{N}=(2,2)$ superspace which has the structure of the super Heisenberg algebra is given by the following two types of deformations 1 :

$$
\begin{aligned}
& *_{Q}=\exp \left[\frac{i}{2} \Theta \epsilon^{\mu \nu} \frac{\overleftarrow{\partial}}{\partial X^{\mu}} \frac{\partial}{\partial X^{\nu}}-\frac{C_{Q}}{2}\left(\overleftarrow{Q_{+}} \overrightarrow{Q_{-}}+\overleftarrow{Q_{-}} \overrightarrow{Q_{+}}\right)\right] \\
& *_{D}=\exp \left[\frac{i}{2} \Theta \epsilon^{\mu \nu} \overleftarrow{\frac{\partial}{\partial X^{\mu}}} \frac{\partial}{\partial X^{\nu}}-\frac{C_{D}}{2}\left(\overleftarrow{D_{+}} \overrightarrow{D_{-}}+\overleftarrow{D_{-}} \overrightarrow{D_{+}}\right)\right]
\end{aligned}
$$

The deformations (33) and (34) are called $Q$-deformation [10] and $D$-deformation [7], respectively. The nontrivial commutation relation in the operator formalism with $Q$-deformation becomes

$$
\begin{aligned}
& {\left[X^{1}, X^{2}\right]=i \Theta-\frac{i}{2} C_{Q} \bar{\theta}^{+} \bar{\theta}^{-}, \quad\left[X^{1}, \theta^{+}\right]=\frac{1}{2} C_{Q} \bar{\theta}^{-}, \quad\left[X^{1}, \theta^{-}\right]=\frac{1}{2} C_{Q} \bar{\theta}^{+},} \\
& {\left[X^{2}, \theta^{+}\right]=\frac{i}{2} C_{Q} \bar{\theta}^{-}, \quad\left[X^{2}, \theta^{-}\right]=-\frac{i}{2} C_{Q} \bar{\theta}^{+}, \quad\left\{\theta^{+}, \theta^{-}\right\}=C_{Q} .}
\end{aligned}
$$

In the case of $D$-deformation, it becomes

$$
\begin{aligned}
& {\left[X^{1}, X^{2}\right]=i \Theta-\frac{i}{2} i C_{D} \bar{\theta}^{+} \bar{\theta}^{-}, \quad\left[X^{1}, \theta^{+}\right]=-\frac{1}{2} C_{D} \bar{\theta}^{-}, \quad\left[X^{1}, \theta^{-}\right]=-\frac{1}{2} C_{D} \bar{\theta}^{+}} \\
& {\left[X^{2}, \theta^{+}\right]=-\frac{i}{2} C_{D} \bar{\theta}^{-}, \quad\left[X^{2}, \theta^{-}\right]=\frac{i}{2} C_{D} \bar{\theta}^{+}, \quad\left\{\theta^{+}, \theta^{-}\right\}=C_{D} .}
\end{aligned}
$$

\footnotetext{
${ }^{1}$ There is another deformation which corresponds to the case of $C_{Q}, C_{D}=0$. However this deformation gives the similar result with $\mathcal{N}=(1,1)$ supertorus. Thus we consider the case of $C_{Q}, C_{D} \neq 0$.
} 
The algebras (35) and (36) can be represented on the module $f\left(s, \eta, \bar{\theta}^{+}, \bar{\theta}^{-}\right)$as

$$
\begin{aligned}
& X^{1} f\left(s, \eta, \bar{\theta}^{+}, \bar{\theta}^{-}\right)=\left(i \frac{\partial}{\partial s} \pm \frac{1}{2} \bar{\theta}^{+} \frac{\partial}{\partial \eta} \pm \frac{1}{2} \bar{\theta}^{-} \eta\right) f\left(s, \eta, \bar{\theta}^{+}, \bar{\theta}^{-}\right), \\
& X^{2} f\left(s, \eta, \bar{\theta}^{+}, \bar{\theta}^{-}\right)=\left(s \mp \frac{i}{2} \bar{\theta}^{+} \frac{\partial}{\partial \eta} \pm \frac{i}{2} \bar{\theta}^{-} \eta\right) f\left(s, \eta, \bar{\theta}^{+}, \bar{\theta}^{-}\right), \\
& \theta^{+} f\left(s, \eta, \bar{\theta}^{+}, \bar{\theta}^{-}\right)=\frac{\partial}{\partial \eta} f\left(s, \eta, \bar{\theta}^{+}, \bar{\theta}^{-}\right), \\
& \theta^{-} f\left(s, \eta, \bar{\theta}^{+}, \bar{\theta}^{-}\right)=\eta f\left(s, \eta, \bar{\theta}^{+}, \bar{\theta}^{-}\right),
\end{aligned}
$$

where the double signs correspond to $Q$ - and $D$-deformation respectively and we set $\Theta=$ $C_{Q}=C_{D}=1$. Note that in the both cases $\bar{\theta}^{+}$and $\bar{\theta}^{-}$belong to the center and the resulting algebra are the super Heisenberg algebra. Supersymmetry is broken to $\mathcal{N}=(1,1)$ in $Q$ deformation [10], but it is unbroken in D-deformation [7].

\subsection{Noncommutative supertori}

First, we consider the construction of noncommutative(NC) supertorus for $\mathcal{N}=(1,1)$ supersymmetry. In order to construct the NC supertorus, we have to get the operators which generate the translation along the cycles of the NC supertorus. However, we do not know how to construct the equations corresponding to (14)-(17) in NC supertorus since we do not have geometrical notion in noncommutative (super)space such as the metric and the disjointed neighborhoods. Thus we construct the generators by simply mimicking the commutative case. In the odd spin structure, The generators of the NC supertorus satisfy

$$
\begin{aligned}
U X^{1} U^{-1} & =X^{1}+1, & U X^{2} U^{-1} & =X^{2}, \\
U \theta U^{-1} & =\theta, & U \bar{\theta} U^{-1} & =\bar{\theta}, \\
V X^{1} V^{-1} & =X^{1}+\operatorname{Re}(\tau+\theta \delta), & V X^{2} V^{-1} & =X^{2}+\operatorname{Im}(\tau+\theta \delta), \\
V \theta V^{-1} & =\theta+\delta, & V \bar{\theta} V^{-1} & =\bar{\theta}+\bar{\delta},
\end{aligned}
$$

which corresponds to (14). Here the supercoordinates $\left(X^{1}, X^{2}, \theta, \bar{\theta}\right)$ satisfy the commutation relation (29). Then the explicit form of the generators $U, V$ is given by

$$
\begin{aligned}
U & =\exp (i s) \\
V & =\exp \left[i(\operatorname{Re} \tau) s+(\operatorname{Im} \tau) \frac{\partial}{\partial s}+\delta \mathcal{Q}+\bar{\delta} \overline{\mathcal{Q}}\right] \\
& =\exp \left[i \operatorname{Re}(\tau+\theta \delta) s+\operatorname{Im}(\tau+\theta \delta) \frac{\partial}{\partial s}+\delta \frac{\partial}{\partial \theta}+\bar{\delta} \frac{\partial}{\partial \bar{\theta}}\right]
\end{aligned}
$$


where $\mathcal{Q}, \overline{\mathcal{Q}}$ are the representation of the supercharges $Q, \bar{Q}$ on the module and are defined by

$$
\mathcal{Q}=\frac{\partial}{\partial \theta}-\frac{i}{2} \theta\left(s-\frac{\partial}{\partial s}\right), \quad \overline{\mathcal{Q}}=\frac{\partial}{\partial \bar{\theta}}-\frac{i}{2} \bar{\theta}\left(s+\frac{\partial}{\partial s}\right)
$$

Here an important comment is in order. Although the generators $U, V$ correctly give the translational property along the cycles, (38), the generator $V$ does not belong to a representation of the super Heisenberg group which is the prerequisite for noncommutative supertorus. In the super Heisenberg group representation of noncommutative superspace, the coordinates play the role of the generators and their (anti)commutators should be constant. However, here $Q, \bar{Q}$ plays the role of $\theta, \bar{\theta}$, and their anticommutator is not constant, which can be seen in (40).

This can be also seen from the embedding map picture. From (39), the embedding map can be written as

$$
\begin{aligned}
& \text { U } \quad \text { V }
\end{aligned}
$$

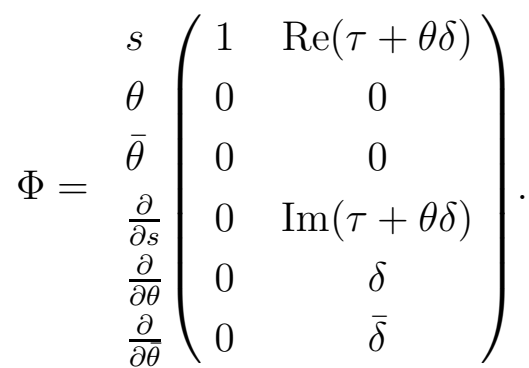

In the embedding map for $V$, we see that the coordinate variable $\theta$ appears. This makes the action of the operator $V$ a lot different from the allowed one given by (11). This is in turn reflected in the commutation relation between $U$ and $V$,

$$
U V=\exp [-i \operatorname{Im}(\tau+\theta \delta)] V U
$$

We now see that it does not satisfy the defining relation for noncommutative torus since $\theta_{i j}$ in (11) fails to be a constant due to the the presence of the coordinate variable $\theta$ in (42).

For the even spin structures, (15)-(17) contain the sign change $\theta \rightarrow-\theta, \bar{\theta} \rightarrow-\bar{\theta}$. Hence in order to construct the generators, we need the generator of sign change for $\theta$ and $\bar{\theta}$, which is given by the spin angular momentum operator

$$
J=\frac{1}{2}\left(\theta \frac{\partial}{\partial \theta}-\bar{\theta} \frac{\partial}{\partial \bar{\theta}}\right)
$$

Then the generators of the NC supertorus with even spin structures are 
- $(+,-)$ structure

$$
\begin{aligned}
& U=\exp (i s) \\
& V=\exp (2 \pi i J) \exp \left[i(\operatorname{Re} \tau) s+(\operatorname{Im} \tau) \frac{\partial}{\partial s}\right]
\end{aligned}
$$

- $(-,+)$ structure

$$
\begin{aligned}
U & =\exp (2 \pi i J) \exp (i s) \\
V & =\exp \left[i(\operatorname{Re} \tau) s+(\operatorname{Im} \tau) \frac{\partial}{\partial s}\right] .
\end{aligned}
$$

- $(-,-)$ structure

$$
\begin{aligned}
& U=\exp (2 \pi i J) \exp (i s) \\
& V=\exp (2 \pi i J) \exp \left[i(\operatorname{Re} \tau) s+(\operatorname{Im} \tau) \frac{\partial}{\partial s}\right]
\end{aligned}
$$

In these three structures, the commutation relation between $U$ and $V$ is given by

$$
U V=\exp (-i \operatorname{Im} \tau) V U
$$

Note that in the above three cases of even spin structures no obstruction appears in the construction of noncommutative supertorus.

Next we consider the $\mathcal{N}=(2,2)$ case. For $\mathcal{N}=(2,2)$ supersymmetry, we proceed in the same manner as in the $\mathcal{N}=(1,1)$ case. For the odd spin structure, the generators $U, V$ satisfy

$$
\begin{array}{lll}
U X^{\mu} U^{-1}=X^{\mu}+e_{U}^{\mu}, & U \theta^{ \pm} U^{-1}=\theta^{ \pm}, & U \bar{\theta}^{ \pm} U^{-1}=\bar{\theta}^{ \pm}, \\
V X^{\mu} V^{-1}=X^{\mu}+e_{V}^{\mu}, & V \theta^{ \pm} V^{-1}=\theta^{ \pm}+\delta^{ \pm}, & V \bar{\theta}^{ \pm} V^{-1}=\bar{\theta}^{ \pm}+\bar{\delta}^{ \pm},
\end{array}
$$

where the supercoordinates satisfy the algebra (37) and the lattice vectors $e_{U}^{\mu}$ and $e_{V}^{\mu}$ are given by

$$
\begin{aligned}
& e_{U}^{\mu}={ }^{t}(1,0), \\
& e_{V}^{\mu}={ }^{t}\left(\operatorname{Re}\left(\tau+\bar{\theta}^{+} \delta^{+}+\theta^{+} \bar{\delta}^{+}\right), \operatorname{Im}\left(\tau+\bar{\theta}^{+} \delta^{+}+\theta^{+} \bar{\delta}^{+}\right)\right) .
\end{aligned}
$$


Then the explicit form of $U, V$ in the $Q$-deformation (33) is given by

$$
\begin{aligned}
U & =\exp (i s) \\
V & =\exp \left[i(\operatorname{Re} \tau) s+(\operatorname{Im} \tau) \frac{\partial}{\partial s}+\delta^{+} \mathcal{Q}_{+}+\delta^{-} \mathcal{Q}_{-}\right] \\
& =\exp \left[i(\operatorname{Re} \tau) s+(\operatorname{Im} \tau) \frac{\partial}{\partial s}+\delta^{+} \eta+\delta^{-} \frac{\partial}{\partial \eta}\right]
\end{aligned}
$$

where the operators $\mathcal{Q}_{ \pm}$are the representation of the supercharges $Q_{ \pm}$on the module, and can be simply represented by

$$
\mathcal{Q}_{+}=\eta \cdot, \quad \mathcal{Q}_{-}=\frac{\partial}{\partial \eta}
$$

In the above we set $\bar{\delta}^{ \pm}=0$, since the representation of $\bar{Q}^{ \pm}$on the module is given by

$$
\overline{\mathcal{Q}}_{+}=\frac{\partial}{\partial \bar{\theta}^{+}}-i \eta\left(s-\frac{\partial}{\partial s}\right), \quad \overline{\mathcal{Q}}_{-}=\frac{\partial}{\partial \bar{\theta}^{-}}-i \frac{\partial}{\partial \eta}\left(s-\frac{\partial}{\partial s}\right),
$$

which contains the second order derivative2, and thus it cannot be included in $V$. The commutation relation between $U$ and $V$ is given by

$$
U V=\exp (-i \operatorname{Im} \tau) V U
$$

and shows no obstruction for noncommutative supertorus. On the other hand, in the $D$ deformation, $U$ and $V$ are obtained as

$$
\begin{gathered}
U=\exp (i s), \\
V=\exp \left[i(\operatorname{Re} \tau) s+(\operatorname{Im} \tau) \frac{\partial}{\partial s}+\delta^{+} \mathcal{Q}_{+}^{\prime}+\delta^{-} \mathcal{Q}_{-}^{\prime}+\bar{\delta}^{+} \overline{\mathcal{Q}}_{+}^{\prime}+\bar{\delta}^{-} \overline{\mathcal{Q}}_{-}^{\prime}\right] \\
=\exp \left[i \operatorname{Re}\left(\tau+2 \bar{\theta}^{+} \delta^{+}\right) s+\operatorname{Im}\left(\tau+2 \bar{\theta}^{+} \delta^{+}\right) \frac{\partial}{\partial s}\right. \\
\left.\quad+\delta^{+} \eta+\delta^{-} \frac{\partial}{\partial \eta}+\bar{\delta}^{+} \frac{\partial}{\partial \bar{\theta}^{+}}+\bar{\delta}^{-} \frac{\partial}{\partial \bar{\theta}^{-}}\right],
\end{gathered}
$$

where the operators $\mathcal{Q}_{ \pm}^{\prime}, \overline{\mathcal{Q}}_{ \pm}^{\prime}$ are the representation of the supercharges $Q_{ \pm}, \bar{Q}_{ \pm}$on the module and are given by

$$
\begin{array}{ll}
\mathcal{Q}_{+}^{\prime}=\eta-i \bar{\theta}^{+}\left(s-\frac{\partial}{\partial s}\right), & \mathcal{Q}_{-}^{\prime}=\frac{\partial}{\partial \eta}-i \bar{\theta}^{-}\left(s+\frac{\partial}{\partial s}\right) \\
\overline{\mathcal{Q}}_{+}^{\prime}=\frac{\partial}{\partial \bar{\theta}^{+}}, & \overline{\mathcal{Q}}_{-}^{\prime}=\frac{\partial}{\partial \bar{\theta}^{-}} .
\end{array}
$$

\footnotetext{
${ }^{2}$ This corresponds to that the supersymmetry generated by $\bar{Q}^{ \pm}$is broken by the $Q$-deformation.
} 
Then the commutation relation between $U$ and $V$ is given by

$$
U V=\exp \left[-i \operatorname{Im}\left(\tau+2 \bar{\theta}^{+} \delta^{+}\right)\right] V U .
$$

Again in this case, we see the obstruction that appeared in the $\mathcal{N}=(1,1)$ case. The $V$ in (54) does not belong to the super Heisenberg group, thus cannot be a generator for noncommutative supertorus.

The embedding maps for both $Q$ - and $D$-deformations can be written from (150) and (54) as

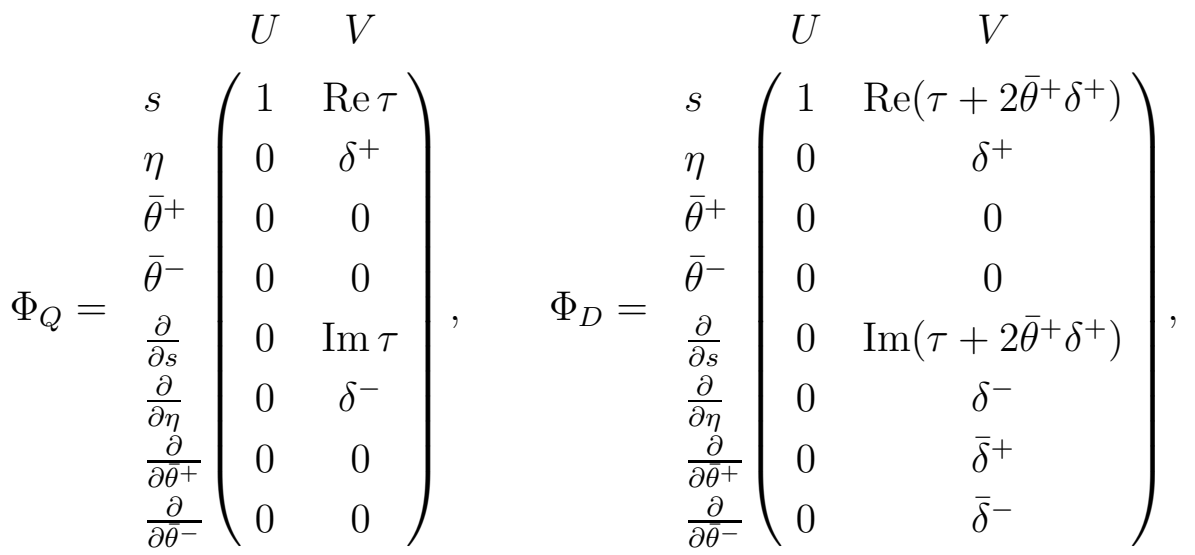

and the embedding map for the $D$-deformation case shows the same problem in the $\mathcal{N}=$ $(1,1)$ case. Therefore, the construction of noncommutative supertorus with odd spin structure in two dimensions is allowed only in the $Q$-deformation with $\mathcal{N}=(2,2)$ supersymmetry.

In the even spin structure cases, there is no obstruction for noncommutative supertorus as in the $\mathcal{N}=(1,1)$ case. To see this, we need the spin operators $J_{\theta}$ and $J_{\bar{\theta}}$ of $\theta^{ \pm}$and $\bar{\theta}^{ \pm}$, respectively, as in the $\mathcal{N}=(1,1)$ case. The explicit form of $J_{\theta}$ and $J_{\bar{\theta}}$ are given by

$$
J_{\theta}=-\frac{1}{2} \theta^{-} \theta^{+}=-\frac{1}{2} \eta \frac{\partial}{\partial \eta}, \quad J_{\bar{\theta}}=\frac{1}{2}\left(\bar{\theta}^{+} \frac{\partial}{\partial \bar{\theta}^{+}}-\bar{\theta}^{-} \frac{\partial}{\partial \bar{\theta}^{-}}\right) .
$$

Then the generators $U$ and $V$ in $(+,-)$ structure for both $Q$ - and $D$-deformations are obtained as

$$
\begin{aligned}
U & =\exp (i s) \\
V & =\exp \left[2 \pi i\left(J_{\theta}+J_{\bar{\theta}}\right)\right] \exp \left[i(\operatorname{Re} \tau) s+(\operatorname{Im} \tau) \frac{\partial}{\partial s}\right] .
\end{aligned}
$$

The generators in the other spin structures can be obtained similarly. The commutation relation between $U$ and $V$ takes the same form with (47). Note that the spin structures of $\theta^{ \pm}$and $\bar{\theta}^{ \pm}$should be the same in order to preserve the algebra (35) and (36). 


\section{Conclusion}

In this paper, we construct noncommutative supertori in two dimensions with $\mathcal{N}=(1,1)$ and $\mathcal{N}=(2,2)$ supersymmetries. In the $\mathcal{N}=(1,1)$ case, only the deformation of the bosonic part is allowed to maintain the supersymmetry and the super Heisenberg group structure. In this case, the super Heisenberg group structure, which is essential to be a noncommutative supertorus, is only maintained for the even spin structures. In the $\mathcal{N}=$ $(2,2)$ case, both bosonic and fermionic parts are deformed with two types of deformations, $Q$ - and $D$-deformation. For the odd spin structure, the generators belong to the super Heisenberg group only in the $Q$-deformation case, in which the supersymmetry is broken down to $\mathcal{N}=(1,1)$. On the other hand, the super Heisenberg group structure is maintained in both $Q$ - and $D$-deformations for the even spin structures. The result shows that for the odd spin structure noncommutative supertorus in two dimensions is allowed only in the $Q$-deformation with $\mathcal{N}=(2,2)$ supersymmetry, while there is no obstruction for the even spin structures. One might understand this result from the underlying property of spin structures: Odd spin structure is related with a translation in the fermionic direction, thus the allowed deformation which is consistent with the super Heisenberg group structure is restricted. Even spin structures are related with translations in the bosonic cycles only, thus they do not interfere with the super Heisenberg group structure.

Next, we comment other aspects of our work. The commutative supertorus was constructed by taking the quotient of the superplane with a proper lattice, and has certain translation properties along its cycles. Noncommutative torus was constructed such that it maintains the properties under the translations along the cycles of commutative torus. We thus construct the generators of the noncommutative supertorus such that the properties under translations along the cycles of the commutative supertorus are maintained.

Noncommutative torus was defined by embedding the lattice into the Heisenberg group, which is equivalent to a central extension of commutative space and represents a deformation of commutative space. The lattice embedding determines how the generators of noncommutative torus act on the module, representing the translations along the cycles. With this in mind, we also identify the embedding maps for noncommutative supertori in two dimensions analyzing the generators of them.

In the super case, we have to additionally implement the spin structures due to the presence of fermionic coordinates. Odd spin structure is realized by implementing the translation in the fermionic direction as in the construction of the noncommutative torus. Even spin structures are realized with appropriate versions of the spin angular momentum operator to express the sign changes of the fermionic coordinates under the translations along the cycles. 


\section{Acknowledgments}

The authors thank KIAS for hospitality during the time that this work was done. This work was supported by the Korea Research Foundation Grants funded by the Korean Government(MOEHRD), KRF-2007-313-C00152 (E. C.-Y.), and KRF-2006-311-C00195 (H. K.), and is the result of research activities (Astrophysical Research Center for the Structure and Evolution of the Cosmos (ARCSEC)) and grant No. R01-2006-000-10965-0 from the Basic Research Program supported by KOSEF (H. N.).

\section{References}

[1] A. Connes, M.R. Douglas, and A. Schwarz, Noncommutative geometry and matrix theory: Compactification on tori, JHEP 9802 (1998) 003 [hep-th/9711162].

[2] A. Connes, Noncommutative geometry (Academic Press, New York, 1994).

[3] J. Polchinski, String theory, vol.1,2 (Cambridge Univ. Press, Cambridge, 1998).

[4] M.B. Green, J.H. Scwarz, and E. Witten, Superstring theory, vol.1,2 (Cambridge Univ. Press, Cambridge, 1987).

[5] N. Seiberg and E. Witten, String theory and noncommutative geometry, JHEP 9909 (1999) 032 hep-th/9908142.

[6] C. S. Chu and P. M. Ho, Noncommutative open string and D-brane, Nucl. Phys. B550 (1999) 151 hep-th/9812219.

[7] D. Klemm, S. Penati, and L. Tamassia, Non(anti)commutative superspace, Class. Quant. Grav. 20 (2003) 2905 arXiv:hep-th/0104190.

[8] H. Ooguri and C. Vafa, The C-deformation of gluino and non-planar diagrams, Adv. Theor. Math. Phys. 7 (2003) 53 [hep-th/0302109]; Gravity induced C-deformation, Adv. Theor. Math. Phys. 7 (2004) 405 hep-th/0303063.

[9] J. de Boer, P. A. Grassi, and P. van Nieuwenhuizen, Non-commutative superspace from string theory, Phys. Lett. B574 (2003) 98 [hep-th/0302078. 
[10] N. Seiberg, Noncommutative superspace, $N=1 / 2$ supersymmetry, field theory and string theory, JHEP 0306 (2003) 010 [hep-th/0305248].

[11] N. Berkovits and N. Seiberg, Superstrings in graviphoton background and $N=1 / 2+$ 3/2 supersymmetry, JHEP 0307 (2003) 010 [hep-th/0306226].

[12] T. Araki, K. Ito, and A. Ohtsuka, Supersymmetric Gauge Theories on Noncommutative Superspace, Phys. Lett. B573 (2003) 209 hep-th/0307076].

[13] S. Ferrara, E. Ivanov, O. Lechtenfeld, E. Sokatchev, and B. Zupnik, Nonanticommutative chiral singlet deformation of $N=(1,1)$ gauge theory, Nucl. Phys. B704 (2005) 154 hep-th/0405049.

[14] K. Ito and H. Nakajima, Non(anti)commutative $N=2$ supersymmetric $U(N)$ gauge theory and deformed instanton equations, Phys. Lett. B629 (2005) 93 arXiv:hep-th/0508052.

[15] A. De Castro, E. Ivanov, O. Lechtenfeld, and L. Quevedo, Non-singlet Q-deformation of the $N=(1,1)$ gauge multiplet in harmonic superspace, Nucl. Phys. B747 (2006) 1 hep-th/0510013; A. De Castro and L. Quevedo, Non-singlet Q-deformed $N=(1,0)$ and $N=(1,1 / 2) U(1)$ actions, Phys. Lett. B639 (2006) 117 [hep-th/0605187.

[16] J. M. Rabin and P. G. O. Freund, Supertori are algebraic curves, Commun. Math. Phys. 114 (1988) 131.

[17] L. Crane and J. M. Rabin, Super Riemann surfaces: Uniformization and Teichmüller theory, Commun. Math. Phys. 113 (1988) 601.

[18] M. Rieffel, Projective modules over higher-dimensional non-commutative tori, Can. J. Math. Vol. XL (1988) 257.

[19] Y. Manin, Theta functions, quantum tori and Heisenberg groups, math.AG/0011197.

[20] Y. Manin, Functional equations for quantum theta functions, math.QA/0307393.

[21] Ee C.-Y. and H. Kim, Quantum thetas on noncommutative $T^{d}$ with general embeddings, J. Phys. A41 (2008) 105201 arXiv:0709.2483.

[22] D. Mumford, Tata lectures on theta III (Birkhauser, Basel-Boston, 1991).

[23] S. Thangavelu, Harmonic analysis on the Heisenberg group (Birkhauser, Boston, 1998). 
[24] J. Rosenberg, A selective history of the Stone-von Neumann theorem, in Operator algebras, quantization, and noncommutative geometry, Contemp. Math. 365, Amer. Math. Soc. (2004).

[25] Ee C.-Y., H. Kim, and H. Nakajima, Noncommutative superspace and super Heisenberg group, JHEP 0804 (2008) 004 arXiv:0711.4663. 